\title{
Optimization of Urea-glycerin Based NADES-UAE for Oxyresveratrol Extraction from Morus alba Roots for Preparation of Skin Whitening Lotion
}

\author{
Tanfidz Alishlah', Abdul Mun'im², Mahdi Jufri'3** \\ ${ }^{1}$ Faculty of Pharmacy Graduate Programme of Herbal Medicine, Universitas Indonesia, Depok, INDONESIA \\ 2Department of Pharmacognosy-Phytochemistry, Faculty of Pharmacy, Universitas Indonesia, Depok, INDONESIA \\ ${ }^{3}$ Faculty of Pharmacy, Laboratory of Pharmaceutical Technology and Drug Development, Universitas Indonesia, Depok, INDONESIA
}

\begin{abstract}
Objective: The oxyresveratrol, polyphenol from Morus alba (Mulberry) roots has been known to have potential skin whitening activities. The application of urea-glycerin-based Natural Deep Eutectic Solvent-Ultrasonic Assisted Extraction (NADES-UAE) method was successful on extraction of polyphenols from plant material. The purpose of this research was to get optimum extraction conditions to enhance oxyresveratrol content and get the best lotion formula based on physical properties evaluation. Methods: The roots were extracted with urea-glycerin-based NADES-UAE method. The NADES-UAE method was employed to determine the optimal extraction condition for two parameters: the molar ratio of urea: glycerin and ultrasonic irradiation time. Oxyresveratrol concentration of the extracts was analyzed with HPLC. Morus alba roots extract was formulated into three formulas of lotion preparation and evaluated based on their physical properties. Results: The optimized conditions for the highest oxyresveratrol concentration from Morus alba roots extract were the molar ratio of urea-glycerin (1:3) and ultrasonic irradiation time as 15 mins. It showed that urea-glycerin UAE method produced Morus alba roots extract with higher
\end{abstract}

oxyresveratrol content than conventional method. Based on physical evaluation from three lotion formulas, formula $A$ was chosen as best formula. Conclusion: This urea-glycerin-based NADES-UAE method was suitable as a green alternative method for the extraction of oxyresveratrol from Morus alba roots because cheaper, faster and easier than conventional extraction method. The lotion preparation which has good physical properties can be applied as a skin whitening cosmetic.

Key words: Natural deep eutectic solvent, Ultrasonic assisted extraction, Urea, Glycerin, Oxyresveratrol, Morus alba, Roots extract, Skin whitening.

Correspondence

Dr. Mahdi Jufri, Laboratory Pharmaceutical Technology and Drug Development, Faculty of Pharmacy, Universitas Indonesia, Depok-16424, INDONESIA.

Phone: +62 81310334771

Email: mahdi.jufri@farmasi.ui.ac.id

DOI: 10.5530/jyp.2019.11.33

\section{INTRODUCTION}

Oxyresveratrol is one of the natural compounds reported to have activity as skin whitening. ${ }^{1}$ The inhibitory activity of tyrosinase is more potent than azelaic acid, curcumin, kojic acid and mimosin. ${ }^{1}$ Oxyresveratrol was reported to inhibit DOPA oxidase activity 150 fold better than resveratrol $^{1}$ and with a concentration of $0.3-5 \mu \mathrm{M}$ showed inhibition of tyrosinase by $25-84 \%$, in which $50 \%$ inhibition was shown at a concentration of $1 \mu \mathrm{M} .{ }^{1}$ Ethanolic extract of roots and stems from Morus alba (mulberry) containing $0-60 \mu \mathrm{g} / \mathrm{ml}$ oxyresveratrol showed inhibition activity of tyrosinase by $0-78 \%$ and $0-62 \% .^{2}$ Methanolic extract of Morus alba roots was reported to have the highest oxyresveratrol content compared to other parts of the plant. ${ }^{3}$ Based on these data, the Morus alba roots can be potential candidates as skin whitening agent.

The common extraction method used to get the oxyresveratrol from Morus alba roots is conventional extraction using organic solvents such as maceration, percolation, reflux and soxhlet. However, this method has some disadvantages such as low extraction efficiency values, much-needed solvents, long extraction time and less environmentally friendly. ${ }^{4}$ Extraction using Natural deep eutectic solvent (NADES) is one of the new green extraction process. Deep eutectic solvent is a mixture of two or more compounds (act as hydrogen bond acceptor and hydrogen bonds donor) which melting point will decrease significantly when mixed compared to their origin melting points. ${ }^{5}$
NADES solvent has an advantage of having physicochemical properties such as low vapor pressure, low toxicity profiles, good mixing with water, biodegradable and environmentally friendly. ${ }^{6}$ Ultrasound-asssisted Extraction (UAE) is one of the most widely method combined with NADES as extraction solvent. UAE method is known to have advantages over other extraction methods, which can increase the extract yield by using fewer solvents and reduce the extraction time. ${ }^{7}$

In the previous study, mulberry root extraction using a conventional method of maceration with variations of organic solvents ethanol and methanol showed low oxyresveratrol content which is around 0.0013 $0.0034 \mathrm{mg} / \mathrm{g}$ dry powder. ${ }^{8}$ The application of urea-glycerin as NADESUAE method was successsful to enhance the polyphenols content in extract from some plant material., ${ }^{910}$ Jeong et al. managed to extract and increase the levels of Epigallocatechin gallate (ECGC) from green tea leaves by using NADES urea-glycerin solvent compared with conventional method. ${ }^{9}$ Therefore, urea-glycerin based NADES-UAE method is expected to enhance the oxyresveratrol content from Morus alba roots. The method of extraction using NADES-UAE is a way that has never been done to obtain the more optimum oxyresveratrol content from Morus alba roots.

An obtained extract from extraction with NADES-UAE can be readily formulated in various forms of cosmetic preparations that act as skin whitening, one of which is a lotion preparation. The lotion is an emulsion 
consisting of more water phases than oil phase and easy to flow. ${ }^{11}$ The main function of the lotion is to moisturize and provide a wetting effect on the skin layer. The lotion is generally preferred in use because of its softer and non-sticky texture. Moreover, it is more readily dispersed on the skin when applied. ${ }^{11}$ The lotion preparation of the Morus alba roots extract should has good physical properties so that can be applied as a skin whitening cosmetic preparation.

\section{MATERIALS AND METHODS}

\section{Study location and duration}

The study was conducted at the Phytochemical Laboratory and Pharmaceutical Technology Laboratory, Faculty of Pharmacy, Universita Indonesia, Depok for March - July 2018.

\section{Materials}

The roots of Morus alba were collected from Rumah Sutera, Bogor, West Java, Indonesia. The roots were washed and dried for six days. The dried roots were ground by using an industrial grinder. The powders were stored in airtight container. Mulberry root powder is also determined by LIPI, Bogor. Oxyresveratrol standard was purchased from Tokyo Chemical Industry Co., Ltd. (TCI) (Japan). Urea, glycerine, propylene glycol, Tween 80, glyceryl monostearate, cethyl alcohol, stearic acid, isopropyl myristate, propyl paraben, methyl paraben, Butyl hidroxy toluene (BHT) were purchased from local supplier. All reagents used for High Performance Liquid Chromatography (HPLC) analysis were HPLC grade and purchased from a local distributor.

\section{Instrumentation}

HPLC system (LC-20AT, Shimadzu, Kyoto, Japan), water bath (Memmert), hot plate magnetic stirer, Ultrasonic chamber, $\mathrm{pH}$ meter (Mettler Toledo) and centrifugator (Sorvall WX Ultra Series WX Ultra 90).

\section{Preparation of NADES urea-glycerin solvent}

Urea-glycerin NADES was prepared by heating and stirring method. The urea and glycerin mixture with a predetermined mass ratio was placed on a Beakerglass. Aquadest was added until volume $20.0 \mathrm{~mL}$ then placed on hotplate magnetic stirrer at $50^{\circ} \mathrm{C}$ with constant stirring of $3000 \mathrm{rpm}$ for 30 mins to form a clear solution.

\section{Optimization of morus alba roots extraction parameters}

Morus alba root powder (1g) was inserted into the test tube, then dissolved in $20 \mathrm{ml}$ of NADES urea : glycerine solution in certain molar ratio 1:1; 1:2 and 1:3 (as parameter described in Table 1). The sample solution was extracted with an ultrasonic chamber according to a specified time 10; 15 and 20 mins (as parameter described in Table 1). The root extract of mulberry was centrifuged and filtered to take its supernatant. The extract was kept in a tightly closed vial as well as shielded by light. The extraction optimization design was shown in Table 1.

\section{Determination of oxyresveratrol standard curves equation}

The oxyresveratrol standard curves equation was determined by the HPLC method. Standard solution was prepared with $1 \mathrm{mg} / \mathrm{mL}$ concentration in $80 \%$ ethanol (1000 ppm). Furthermore, working standard solutions were diluted with concentrations of $3.125 \mathrm{ppm}$ to $100 \mathrm{ppm}$.

\section{Determination of oxyresveratrol by HPLC}

The content of oxyresveratrol in the Morus alba roots extracts were determined by HPLC method. The HPLC analysis was used $\mathrm{C}_{18}$ bondedsilica gel column (YMC, $5 \mu \mathrm{m}, 150 \times 4.6 \mathrm{~mm}$, Phenomenex, Torrance,
Table 1: The extraction optimization design.

\begin{tabular}{ccc} 
Run & Urea : glycerin ratio (molar) & Time (minutes) \\
\hline 1 & $1: 1$ & 10 \\
2 & $1: 1$ & 15 \\
3 & $1: 1$ & 20 \\
4 & $1: 2$ & 10 \\
5 & $1: 2$ & 15 \\
6 & $1: 2$ & 20 \\
7 & $1: 3$ & 10 \\
8 & $1: 3$ & 15 \\
9 & $1: 3$ & 20 \\
\hline
\end{tabular}

Table 2: The Formula of Whitening Skin Lotion.

\begin{tabular}{cccc}
\hline Material & Formula A & Formula B & Formula C \\
\hline & \multicolumn{3}{c}{ Concentration (\%) } \\
\hline M. alba roots extract & $35 \%$ & $35 \%$ & $35 \%$ \\
Cetyl alcohol & $2 \%$ & $4 \%$ & $6 \%$ \\
Stearic acid & $1 \%$ & $1 \%$ & $1 \%$ \\
Isppropyl miristate (IPM) & $5 \%$ & $5 \%$ & $5 \%$ \\
Tween 80 & $3.88 \%$ & $4.13 \%$ & $4.29 \%$ \\
Glyceril monostearate (GMS) & $1.12 \%$ & $0.87 \%$ & $0.71 \%$ \\
Propylen glycol & $15 \%$ & $15 \%$ & $15 \%$ \\
Metylr>araben & $0.18 \%$ & $0.18 \%$ & $0.18 \%$ \\
Propyl paraben & $0.02 \%$ & $0.02 \%$ & $0.02 \%$ \\
Butyl hidroxy toluene (BHT) & $0.5 \%$ & $0.5 \%$ & $0.5 \%$ \\
Aquadest & ad 100 & ad 100 & ad 100 \\
\hline
\end{tabular}

USA) and system (LC-20AT, Shimadzu, Kyoto, Japan), equipped with a UV-Vis detector (SPD-20A, Shimadzu, Japan) in the isocratic mode. The mobile phase was used acetonitrile: $1 \%$ acetic acid solution with ratio $25: 75$. The flow rate was $1 \mathrm{ml} / \mathrm{min}$ and runtime set for $10 \mathrm{~min}$. UV detector wavelength was $320 \mathrm{~nm}$ and the injection volume was $20 \mu \mathrm{l} .^{12}$ The oxyresveratrol content was calculated as follows

Oxyresveratrol content $=\frac{\text { sample area }}{\text { standard area }} \times \frac{\text { standard volume injected }}{\text { sample volume injected }} \times \mathrm{C}$ standard

\section{Formulation of Skin Whitening Lotion}

The three formulas of skin whitening lotion were shown in Table 2.

\section{Preparation of Skin Whitening Lotion}

All the ingredients were weighed using formulations as described in Table 2. Cethyl alcohol, stearic acid, isopropyl myristate, glyceril monostearate and $\mathrm{BHT}$ were placed into a porcelain cup, heated at $60-70^{\circ} \mathrm{C}$ in a waterbath, then were stirred homogeneously (Oil mixture).

Methyl paraben and propyl paraben were dissolved with propylene glycol. Tween 80 , a mixture of propylene glycol-preservatives and aquadest were added into a Beakerglass and were homogeneously heated at $60-70^{\circ} \mathrm{C}$ in a waterbath (Aqueous mixture). The oil mixture and aqueous mixture were mixed at $\pm 70^{\circ} \mathrm{C}$ and homogenized with homogenizer at $600-850 \mathrm{rpm}$ until lotion mass was formed. After the base of lotion finished, the Morus alba roots extract was added into the base and homogenized with homogenizer at 300-600 rpm. The lotion was inserted into the cosmetic 
bottle. The final dosage evaluations (Organoleptic observation, Homogeneity testing, $\mathrm{pH}$ testing and viscosity testing) were performed.

\section{RESULTS}

\section{The result of morus alba roots extraction}

The dried powder of Morus alba roots occured as light brown powder as shown in Figure 1. The extract from Morus alba roots powder using NADES urea glycerin-UAE on pilot scale was shown in Figure 1. The Morus alba extract occured as brown liquid with spesific odor.

\section{Determination of oxyresveratrol standard curves equation}

The six working standard solutions were prepared with concentrations of $3.125 \mathrm{ppm}$ to $100 \mathrm{ppm}$. The oxyresveratrol standard curves shown in Figure 2 with equation, $y=97402 x+61597$ and $R$ value 0.997 .

\section{Optimization of morus alba roots extraction parameters}

The 9 runs of two different parameters were studied and the result for oxyresveratrol content from Morus alba roots extract was analyzed by HPLC was shown in Table 3. The highest oxyresveratrol content was achieved in run 8 with 1: 3 urea-glycerin ratio and 15 mins extraction time, which was 121.168 ppm of oxyresveratrol. Based on calculation, Morus alba roots extract in run 8 contains oxyresveratrol of $2.42 \mathrm{mg} / \mathrm{g}$ dry powder. The comparison of chromatogram between standard and sample were shown in Figure 3.

\section{Formulation of skin whitening lotion}

Extract of Morus alba roots was formulated into a dosage lotion as skin whitening. The lotion base consists of two phases: oil phase and aqueous
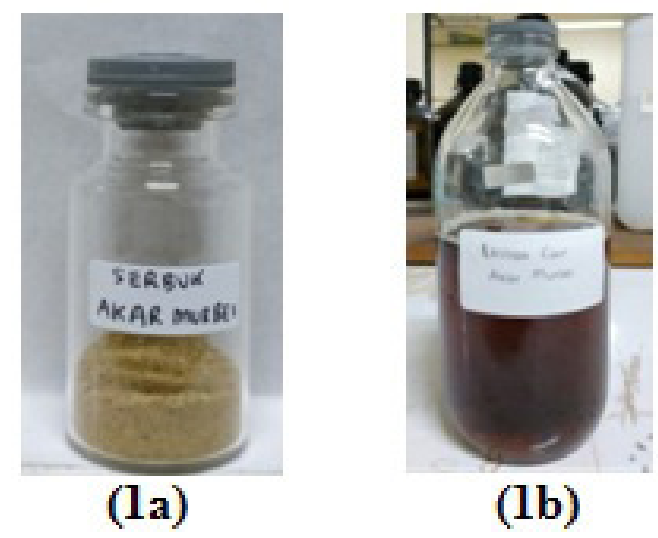

Figure 1: (a) The dried powder of M. alba roots; (b) The liquid extract of M. alba roots.

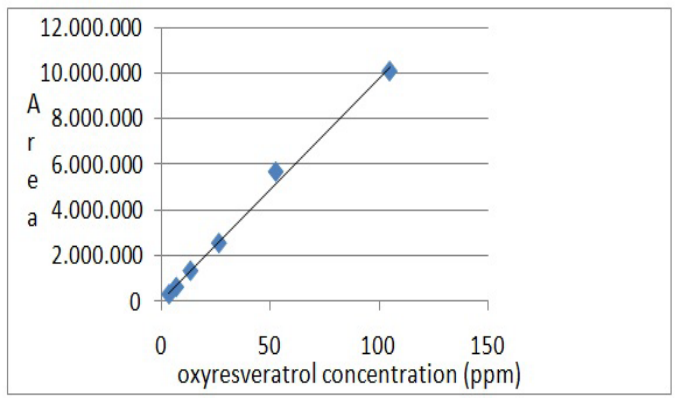

Figure 2: The oxyresveratrol standard curves by HPLC.
Table 3: Oxyresveratrol concentration in $M$. alba roots extract analyzed by HPLC.

\begin{tabular}{cccccc}
\hline Run & $\begin{array}{c}\text { Urea:glycerin } \\
\text { ratio (molar) }\end{array}$ & $\begin{array}{c}\text { Time } \\
(\mathrm{min})\end{array}$ & Area & $\begin{array}{c}\text { Oxyresveratrol } \\
\text { content }(\mathrm{ppm})\end{array}$ & $\begin{array}{c}\text { Retention } \\
\text { time }\end{array}$ \\
\hline 1 & $1: 1$ & 10 & 6352204 & 64.584 & 8.157 \\
2 & $1: 1$ & 15 & 6711622 & 68.274 & 8.133 \\
3 & $1: 1$ & 20 & 7160866 & 72.886 & 8.159 \\
4 & $1: 2$ & 10 & 9027371 & 92.049 & 8.107 \\
5 & $1: 2$ & 15 & 9258573 & 94.423 & 8.153 \\
6 & $1: 2$ & 20 & 10088530 & 102.944 & 8.074 \\
7 & $1: 3$ & 10 & 9969095 & 101.718 & 8.164 \\
$\mathbf{8}$ & $\mathbf{1 : 3}$ & $\mathbf{1 5}$ & $\mathbf{1 1 8 6 3 6 1 6}$ & $\mathbf{1 2 1 . 1 6 8}$ & $\mathbf{8 . 1 3 9}$ \\
9 & $1: 3$ & 20 & 11047707 & 112791 & 8.148 \\
\hline
\end{tabular}
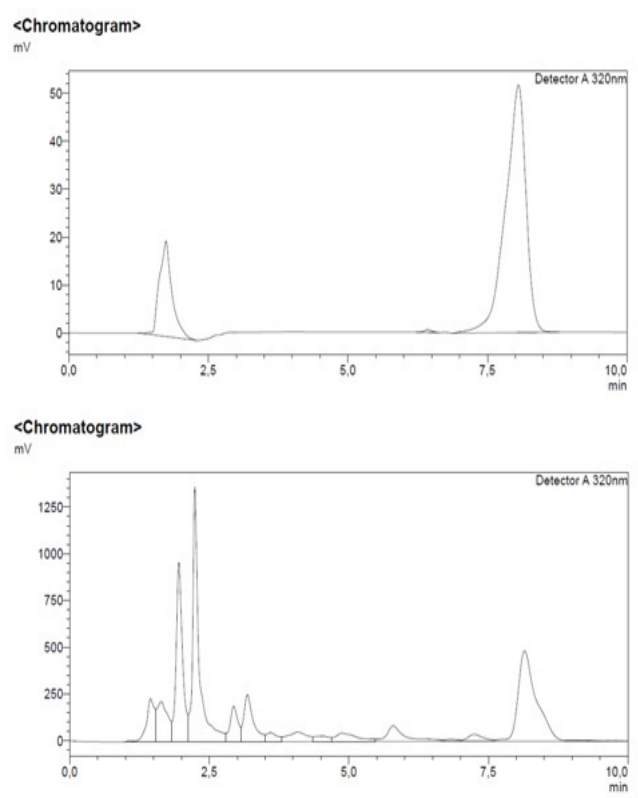

Figure 3: HPLC Chromatograms of standard oxyresveratrol (above) and sample NADES-UAE mulberry root extract (below).

phase can be mixed in the presence additional emulsifier. The desired lotion is oil in water $(\mathrm{o} / \mathrm{w})$ type lotion. Three formulas of lotion were prepared and was performed physic evaluation. The result of physic evaluation shown in Figure 4, Figure 5 and Table 4.

\section{DISCUSSION}

Oxyresveratrol is a substituted stilbene compound of hydroxyl groups, found mostly in roots, stems, leaves and plant fruits of the Moraceae family, Liliaceae and Gnetaceae. ${ }^{13,14}$ Based on study above, NADES ureaglycerin was used as extractor solvent for Morus alba roots. Urea acts as hydrogen bond acceptor (HBA) and glycerin act as hydrogen bond donor (HBD). ${ }^{9}$ In NADES preparation, hydrogen bonding formed due to the interaction of intermolecular interaction and the intramolecular hydrogen bonds. ${ }^{15}$ Extraction from Morus alba roots with urea-glycerin was able to get the optimum oxyresveratrol. The ability of NADES solvent urea-glycerine in extracting oxyresveratrol is due to the similarity of polarity between oxyresveratrol and solvent. Oxyresveratrol is semipolar polyphenolic compound with coefficient of partition $(\log P) 2.55 .{ }^{16}$ Glycerin as sugar alcohol has lower polarity if compared to organic acid based-DES, amino acid based-DES and sugar based-DES which 


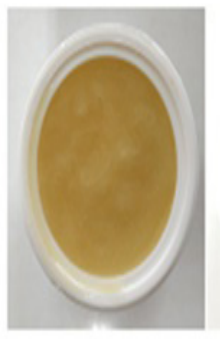

Formula A

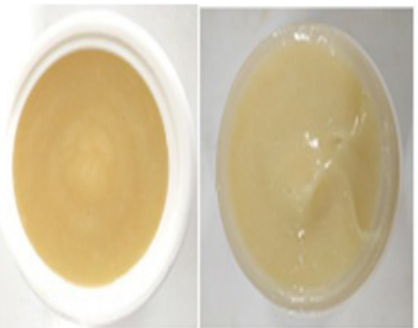

Formula B Formula C

Figure 4: Visual organoleptic of M. alba roots lotion (Formula A; Formula B; Formula C).

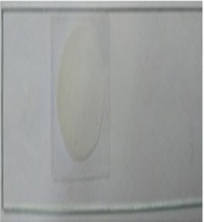

Fomula

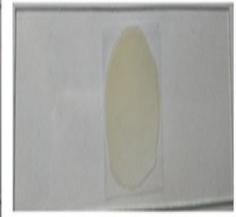

Formula B

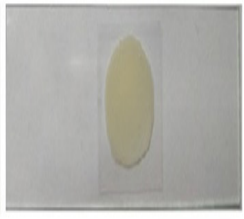

Formula C
Figure 5: Homogeneity testing result of $M$. alba roots lotion (Formula $A$; Formula B; Formula C).

Table 4: The results of physical evaluation of $M$. alba roots lotion

\begin{tabular}{|c|c|c|c|c|}
\hline Formula & Organoleptic & $\mathrm{pH}$ & $\begin{array}{c}\text { Viscosity } \\
\text { (centi poise) }\end{array}$ & Homogeneity \\
\hline A & $\begin{array}{c}\text { Color : light brown } \\
\text { Odor : aromatic } \\
\text { Feel when applied : not } \\
\text { sticky, no white cast }\end{array}$ & 6.12 & $2426.9 \mathrm{Cp}$ & Homogeneous \\
\hline B & $\begin{array}{c}\text { Color : light brown } \\
\text { Odor : aromatic } \\
\text { Feel when applied : not } \\
\text { sticky, white cast }\end{array}$ & 6.28 & $4261.2 \mathrm{Cp}$ & Homogeneous \\
\hline $\mathrm{C}$ & $\begin{array}{c}\text { Color : light brown } \\
\text { Odor : aromatic } \\
\text { Feel when applied : } \\
\text { slightly sticky, white cast }\end{array}$ & 6.35 & $6253.8 \mathrm{Cp}$ & Homogeneous \\
\hline
\end{tabular}

their polarity are very close to water or the most polar organic solvents (Methanol, ethanol, etc). ${ }^{5}$ On previous study, research by Jeong et al. also managed to extract and increase the levels of Epigallocatechin gallate (ECGC) and polyphenol compounds contained in green tea leaves by using NADES urea-glycerin solvent. ${ }^{9}$ Furthermore, the use of NADES urea and glycerin for extraction has the advantage of having a shorter extraction stage because the solvent does not need to be evaporated or removed as in conventional extraction. In addition, urea and glycerin solvents used in the extraction can be applied directly in topical preparations when formulated because urea and glycerin are common excipients as humectant or wetting agent used in cosmetic products to keep moisture in the product during storage.

Based on study, the Morus alba roots extract using urea-glycerin with ratio 1:3 has the highest oxyresveratrol content than other conditions. Urea-glycerine with ratio 1:3 and aquadest addition could affect the viscosity and polarity of solvent. The higherglycerine amount used in solvent the more increase the viscosity, furthermore the addition of water could adjust the property of NADES. Water addition could also enhance solvent polarity. ${ }^{17}$ The use of urea-glycerine with ratio $1: 3$ with water addition until $20 \mathrm{~mL}$ could have the most similar polarity with oxyresveratrol as target compound. However, an excessive water addition can also break the hydrogen bond between HBD and HBA and decrease the interactions between the NADES and target compounds. ${ }^{17}$ Therefore a suitable viscosity and polarity of solvent can improve the extraction yields significantly.

One of criteria for green extraction method is to reduce energy consumption by using innovative technology such as UAE. ${ }^{18} \mathrm{UAE}$ has been chosen as extraction method due to minimalize thermal degradation and oxidation from target compound. ${ }^{19}$ Oxyresveratrol as antioxidant can easily oxydized therefore UAE method is more suitable for extraction. UAE capability to enhance the extraction yield is mainly due to cavitation bubbles in the solvent produced by the ultrasonic wave passage causing microjet impacts. Furthermore, shockwave-induced damage of plant cell wall causing releasing of cell content into the solvent. ${ }^{20}$

On previous study, UAE method was used in the extraction of phenolic compounds of Carthamus tinctorius and Chamaecyparis obtuse by using different DES and extraction efficiency was notimproved in comparison to conventional extraction method. The possible reason for such a low productivity of UAE could be due to extraction temperature because in previous studies UAE was conducted at room temperature. ${ }^{21,22}$ Therefore, in our study the extraction process using UAE with temperature $50^{\circ} \mathrm{C}$. Furthermore, the temperature of extraction with UAE relying on ultrasonic waves that can cause heat effects. The temperature is depend on time extraction. The increase in temperature can occur when the extraction time is longer used. ${ }^{23}$ In this study, the optimum extraction time to get the highest oxyresveratrol concentration was 15 mins. The oxyresveratrol concentration decrease when time extraction become 20 mins. It could be happened due to the compound is sensitive to high temperature that affected degradation of the compound. ${ }^{24}$ Therefore heat is used in oxyresveratrol extraction needs to be controlled.

Morus alba roots extract in run 8 with urea-glycerin UAE method contains oxyresveratrol of $2.42 \mathrm{mg} / \mathrm{g}$ dry powder. In the previous study, mulberry root extraction using a conventional method of maceration with variations of organic solvents ethanol and methanol showed low oxyresveratrol content which is only $0.0013-0.0034 \mathrm{mg} / \mathrm{g}$ dry powder. ${ }^{8}$ It showed that urea-glycerin UAE method produced Morus alba roots extract with higher oxyresveratrol content than conventional method.

If compared with IL-MAE method as modern extraction method, oxyresveratrol content with urea-glycerin UAE method is lower. The oxyresveratrol content using IL-MAE method for oxyresveratrol extraction from Morus alba roots was $7.016 \mathrm{mg} / \mathrm{g}$ dry powder. ${ }^{25}$ Despite that, the disadvantages of IL-MAE that they cannot be hindered are high price, water stability, high toxicity, very low atom economy for their synthesis, difficulty in purifying and poor biodegradability. ${ }^{5}$ Considering that, developing NADES as a new green and efficient solvents for oxyresveratrol extraction from Morus alba roots is one of effective alternative.

The use of NADES urea and glycerin for extraction has the advantage of having a shorter extraction stage because the solvent does not need to be evaporated or removed as in conventional extraction. In addition, urea and glycerine solvents used in the extraction can be applied directly in topical preparations when formulated because urea and glycerine are common excipients used in cosmetic products. ${ }^{9,26}$

After we get the optimum extraction condition, the Morus alba extract could be readily applied into skin whitening lotion formula. We have three formulas which have different cethyl alcohol concentration as lipid base. The concentration of lipid material is something matter during 
lotion formulation due to the lotion need to be smooth, not sticky and has a good spreadability when applied on skin. The other materials have the same concentration between three formulas.

Some materials have been chosen as skin whitening lotion formula. Cethyl alcohol act as lipid base which form a base lotion. ${ }^{26}$ Isopropilmyristate act as non greasy emollient that is absorbed easily by the skin. ${ }^{26}$ A combination of emulsifying agents (Tween 80 and glyceryl monostearate) is used to obtain a more stable emulsion. Propylene glycol acts as penetration enhancer because active compound need to penetrate into the stratum basale of epidermis which melanocyte cells are found as a targetting cells of skin whitening product. Propylene glycol can disrupt the horny layer of structured lipids of the skin. ${ }^{27} \mathrm{BHT}$ as antioxidants was added because the formulation contains lipid materials that are easily oxidized. ${ }^{26}$ Methyl paraben and propyl paraben were added because herbal ingredients used in the preparation are easily overgrown with bacteria. Methyl paraben and propyl paraben are broad-spectrum preservatives and active in a wide $\mathrm{pH}$ range. ${ }^{26}$ Combination of methyl paraben and propyl paraben increased the affectivity of preservatives. ${ }^{26}$

On this study, the best formula based on organoleptic visual was Formula A which has light brown color, aromatic odor, not sticky and no white cast when applied on skin. Based on $\mathrm{pH}$ testing, the best formulation which has the most suitable $\mathrm{pH}$ was Formula A with $\mathrm{pH}$ 6.12, which not too close to upper limit of skin $\mathrm{pH}$ range $4.5-6.5$. The $\mathrm{pH}$ value should not be too acidic as it may cause irritation, while the alkaline $\mathrm{pH}$ can cause dry skin. ${ }^{28}$ Based on homogenity testing, all formulas showed the homogenous lotions, there is no particle seen on the plate glass (Figure 5). Based on viscosity testing, the best formula was Formula A with viscosity 2426.9 Cp. Purwaningsih. showed that skin lotion with viscosity about $2500 \mathrm{Cp}$ was the most acceptable during sensory et al. characteristic testing and spread easily when applied on skin..$^{29}$ Based on physical evaluation, Formula A was chosen as best formula of Morus alba roots skin whitening lotion.

\section{CONCLUSION}

The optimized conditions for the highest oxyresveratrol concentration from Morus alba roots were as follows: the molar ratio of urea-glycerin (1:3) and ultrasonic irradiation time 15 mins. It showed that ureaglycerin UAE method produced Morus alba roots extract with higher oxyresveratrol content than conventional method. This urea-glycerinbased NADES-UAE method was suitable as agreen alternative method because cheaper, faster and easier than conventional extraction method. The NADES solvent also environmentally friendly and less toxicity for human. Moreover, the extract could be readily applicable to cosmetic or pharmaceutical formulations for skin without solvent removal, so that it can reduce the production stage. The formula A of lotion preparation has good physical properties so that can be applied as a skin whitening cosmetic. Furthermore, the future research has to be followed for more investigation.

\section{ACKNOWLEDGEMENT}

This study was supported by Universitas Indonesia for Hibah PITTA 2018.

\section{CONFLICT OF INTEREST}

The authors declare no conflict of interest.

\section{ABBREVIATIONS}

NADES: Natural deep eutectic solvent; DES: Deep eutectic solvent; UAE: Ultrasound-assisted extraction; HPLC: High-performance liquid chromatography; HBA: Hydrogen bond acceptor; HBD: Hydrogen bond donor; BHT: Butylated hydroxy toluena.

\section{REFERENCES}

1. Shin NH, Ryu SY, Choi EJ, Kang SH, Chang IM, Min KR, et al. Oxyresveratrol as the Potent Inhibitor of Dopa Oxidase Activity of Mushroom Tyrosinase. J Biochem Bioph Res Commun.1998;243(3):801-3

2. Chang LW, Juang LJ, Wang BS, Wang MY, Tai HM, Hung WJ, et al. Antioxidant and Antityrosinase Activity of Mulberry (Morus alba L) Twigs and Root Bark. Food and Chemical Technology. 2011;49(4):785-90.

3. Ayinampudi SR, Wang Y, Avula B, Smillie TJ, Khan IA. Quantitative Analysis of Oxyresveratrol in Different Plant Parts of Morus Species and Related Genera by HPTLC and HPLC. Journal of Planar Chromatography. 2011;24(2):125-9.

4. Sarker S, Latif Z, Gray A. Natural Products Isolation. Totowa: Humana Press. 2015.

5. Ruesgas-Ramón M, Figueroa-Espinoza MC, Durand E. Application of Deep Eutectic Solvents (DES) for Phenolic Compounds Extraction: Overview, Challenges and Opportunities. Journal of Agricultural and Food Chemistry. 2017;65(18):3591-601.

6. Dai Y, Witkamp GJ, Verpoorte R, Choi YH. Natural Deep Eutectic Solvents as a New Extraction Media for Phenolic Metabolites in Carthamus tinctorius L. Analytical Chemical. 2013;85(13):6272-8

7. Panja P. Green extraction methods of food polyphenols from vegetable materials. Food Science. 2017;1-10.

8. Faizatun AE, Djajadisasra J, Mardliyati E. The Study of Antioxidant and Antityrosinase Activity of Extract from Mulberry Root (Morus alba L). J Pharm Sci and Res. 2017;9(11):2004-8.

9. Jeong KM, Ko JY, Zhao J, Jin Y, Yoo DE, Han SY, et al. Multi-functioning deep eutectic solvents as extraction and storage media for bioactive natural products that are readily applicable to cosmetic products. Journal of Cleaner Production. 2017; 151:87-95.

10. Bakirtzi C, Triantafyllidou K, Makris DP. Novel lactic acid-based natural deep eutectic solvents: Efficiency in the ultrasound-assisted extraction of antioxidant polyphenols from common native Greek medicinal plants. Journal of Applied Research on Medicinal and Aromatic Plants. 2016;3(3):120-7.

11. Mitsui T. New Cosmetic Science. Amsterdam: Elsevier. 1998.

12. Soonthornsit N, Pitaksutheepong C, Hemstapat W, Utaisincharoen $P_{1}$ Pitaksuteepong T. In vitro Anti-Inflammatory Activity of Morus alba L. Stem Extract in LPS-Stimulated RAW 264.7 Cells. Evid-Based Comp and Alt Med. 2017.

13. Xiong Y, GuoDA, Huang HL. Chemical constituents from tubers of Smilax china L. Modern Chinese Med. 2008;10:20-2.

14. Ti HH, Wu P, Lin LD. Stilbenes and flavonoids from Artocarpus nitidus subspling nanensis. Fitoterapia. 2011;82(4):662-5.

15. Zhekenov T, Toksanbayev N, Kazakbayeva Z, Shah D, Mjalli FS. Formation of Type III Deep Eutectic Solvents and Effect of Water on Their Intermolecular Interactions. Fluid Phase Equilibria. 2017;441:43-8.

16. European Bioinformatics Institute. ChemBL Database. European Molecular Biology Laboratory [cited 2018 July 20]. Available from: https://www.ebi.ac.uk/ chembldb/compound/inspect/CHEMBL43065.

17. Peng $X$, Duan MH, Yao XH, Zhang YH, Zhao CJ, Zu YG, Yu JF et al.Green extraction of five target phenolic acids from Lonicerae japonicae Flos with deep eutectic solvent. Separation Purifiation Technology. 2016;157:249-57.

18. Chemat F, Vian MA, Cravotto G. Green Extraction of Natural Products: Concept and Principles. International Journal of Molecular Sciences. 2012;13(7):8615-27.

19. Bubalo MC, Vidovic S, Redovnikovic IR, Jokic S. Green Solvents for Green Technologies. Journal of Chemical Technology and Biotechnology. 2015;90(9):1631-9.

20. Esclapez MD, García-Pérez JV, Mulet A, Cárcel JA. Ultrasound assisted extraction of natural products. Food Engineering Reviews. 2011;3(2):108-20.

21. Bi W, Tian M, Row KH. Evaluation of alcohol-based deep eutectic solvent in extraction and determination of flavonoids with response surface methodology optimization. Journal of Chromatography A. 2013;1285:22-30.

22. DaiY, Witkamp GJ, Verpoorte R, Choi YH. Natural deep eutectic solvents as new extraction media for phenolic metabolites in Carthamus tinctorius L. Analytical Chemistry. 2013;85(13):6272-8.

23. Legay M, Nicolas G, SthephaneLP, Primius B, Bontemps A. Enhancement of Heat Transfer by Ultrasound : Review and Recent Advances. International Journal of Chemical Engineering. 2011;1-17.

24. Guaman-Balcazar MC, Setyaningsih W, Palma M, Barroso CG. Ultrasoundassisted Extraction of Resveratrol from Functional Foods: Cookies and Jams. Applied Acoustics. 2016;103:207-13

25. Fadhilah M, Mun'im A, Mahdi J. Ionic Liquid-based Microwave-Assisted Extraction (IL-MAE) of Oxyresveratrol from Morus alba Roots. Journal of Applied Pharmaceutical Science. 2018;8(06):008-13.

26. Rowe RC, Sheskey PJ, Quinn ME. Handbook of Pharmaceutical Excipients (6 $6^{\text {th }}$ ed.). London: Pharmaceutical Press. 2009. 
27. Wathoni N, Sriwidodo, Luhur MP. Effect of iontophoresis and propylene glycol on the in vitro diffusion of ethyl vitamin C cream. Int Res J Pharm App Sci. 2012;2(4):31-4.

28. Levin J, Maibach HI. Human Skin Buffering Capacity: An Overview. New York:
Marcel Dekker, Inc. 2007.

29. Purwaningsih S, Salamah E, Budiarti TA. Formulation of Skin Lotion with Carragenan Addition and Natural Antioxidant from Rhizophora mucronata Lamk. Jurnal Akuatika. 2014;5(1)

Article History: Submission Date : 10-10-2018; Revised Date : 30-11-2018; Acceptance Date : 26-01-2019.

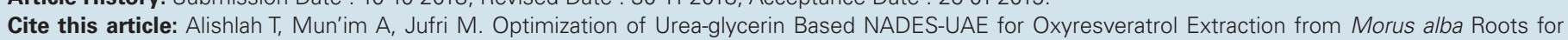

Preparation of Skin Whitening Lotion. J Young Pharm. 2019;11(2):155-60. 